riccafd

Revista Iberoamericana de Ciencias de la Actividad Física y el Deporte

\title{
LA ALTERNATIVA DE LOS EXERGAMES EN LA MEJORA DE LA CALIDAD DE VIDA DE LA TERCERA EDAD
}

THE ALTERNATIVE OF EXERGAMES IN IMPROVING THE QUALITY OF LIFE OF THE ELDERLY

\section{Cabrera-Fernández, Á ${ }^{1}$; Chacón-Cuberos, $\mathrm{R}^{1}$ y Castro-Sánchez, $\mathrm{M}^{2}$}

1 Grupo de investigación HUM-238. Universidad de Granada (España) cabrerafdezangelGrD@correo.ugr.es ; ramonchaconcuberos@ugr.es

${ }^{2}$ Profesor Universidad Santo Tomás (Chile) manue87@correo.ugr.es

Código UNESCO: 2312 Salud Pública

Clasificación Consejo de Europa: 17 Otras: gerontología y Deporte

Recibido el 14 de junio de 2015

Aceptado el 2 de octubre de 2015

Correspondencia: Manuel Castro Sánchez: manue87@correo.ugr.es

\section{RESUMEN}

Actualmente vivimos en una sociedad claramente condicionada por el alcance de las nuevas tecnologías. Asimismo, el significativo aumento del número de nuestros mayores constituye otra característica que la determina. Esta situación plantea la necesidad de propuestas que promuevan la calidad de vida y bienestar en la tercera edad. Para ello, se ha realizado una revisión bibliográfica considerando el ámbito de la actividad física, el cual se concretada como una de las bazas más importantes a tratar para lograr ese objetivo; y el papel que pueden jugar las nuevas tecnologías en él, concretamente los «Exergames». Por ello, y con el fin de buscar alternativas para la mejora de la salud en la tercera edad, este estudio pretende poner en relieve la utilización de las nuevas tecnologías con dicha meta. En este sentido, se destaca el uso 
de los videojuegos activos, una tecnología que lleva en auge desde hace algún tiempo, con la cual nuestros mayores podrán no solo mejorar su condición física y mental, sino su calidad de vida de forma global.

\section{PALABRAS CLAVE}

Actividad Física, Exergames, Salud, Tercera Edad.

\section{ABSTRACT}

We now live in a society that is clearly conditioned by the development of new technologies. Although, we must also point out another feature of our society: the increasing number of our elders, due to numerous factors. What comes to claim a proposals that promotes a good quality of life for seniors. To do this, it has become a literature review, reviewing in one hand physical activity, considered one of the most important assets to try to achieve that end; and secondly, the potential role of new technologies, specifically the "Exergames". On the occasion of proposing it as an alternative to improve the quality of life of our seniors, this research aims to raise awareness on how to use new technologies. Although within them we include "Exergames" leading technology booming for some time, with which our seniors will not only improve your physical and mental condition and thereby improve their quality of life.

\section{KEYWORDS}

Physical Activity, Exergames, Elderly, Healthy.

\section{INTRODUCCIÓN}

El continuo desarrollo tecnológico vivido en las últimas décadas ha determinado la configuración de la sociedad de hoy en dia ${ }^{1}$. De hecho, se podría decir que una de las características principales que posee el entramado 
social actual es el papel que juega la tecnología, pues a través de ella podemos intercomunicarnos e intercambiar grandes cantidades de información en muy poco tiempo. Sin embargo, no podemos concretar el énfasis tecnológico de los últimos años como la única característica de nuestra sociedad, sino que existen otros factores políticos, económicos o sociodemográficos que destacan; como es el creciente aumento del número de ancianos que forman parte de ella ${ }^{2,3}$.

Centrándonos en esta última característica, podemos decir que son numerosos los aspectos que indican el incremento de población mayor de 60 años. En esta línea, estudios como el de Carone y Costello ${ }^{4}$, vaticinaron el inminente crecimiento de la población correspondiente al a tercera edad en la Unión Europea, debido principalmente a factores demográficos. Primero, es evidente destacar que las tasas de fecundidad son y seguirán siendo menores que la tasa de reemplazo natural de la población. Segundo, el reciente descenso de la tasa de fecundidad, tras la explosión demográfica de posguerra y la inminente jubilación de dicha generación, aumentará la tasa de dependencia durante varias décadas. Tercero, otro dato de evidencia pronunciada es que la esperanza de vida se incrementó en ocho años desde 1960, dando como resultado el incremento en seis años más para los hombres y cinco para las mujeres para 2050. Cuarto, se proyecta que pese al aporte que supone la inmigración, no bastará para compensar la baja fecundidad y la creciente esperanza de vida $2,4,5$.

En este sentido, resulta fundamental la concreción de programas e iniciativas que permitan el cuidado y mejora de la calidad de vida de la tercera edad. Por ello, la práctica de actividad física es considerada como una de las mejores herramientas para mejorar la salud y prevenir numerosas enfermedades de distinta índole ${ }^{3,6}$. Sin embargo, muchos de nuestros mayores siguen estilos de vida notoriamente sedentarios, debido en múltiples casos a prejuicios preconcebidos hacia la realización de ejercicio. Otro aspecto a destacar es la brecha digital existente en la tercera $\operatorname{edad}^{7}$, pues es bien sabido que la mayoría de nuestros ancianos no utilizan las nuevas tecnologías que gobiernan el día a 
día. Y es que, según Sevilla et al. ${ }^{8}$, la tendencia del uso de las TIC va en un aumento constante, aunado las innovaciones tecnológicas y los servicios de comunicación e internet que ofrecen los medios. Son, en definitiva, una poderosa herramienta que facilita la información, comunicación y fortalece el desarrollo de habilidades y nuevas formas de construcción del conocimiento ${ }^{7}$.

Resulta evidente que el sector de población perteneciente a la tercera edad pueda valerse de la gran expansión tecnológica de la sociedad actual. Esta premisa es perseguida por varios estudios ${ }^{6,9,10}$, los cuales, destacan el interés existente en los adultos mayores hacia los videojuegos que promueven el ejercicio físico y el bienestar. De este modo, y a pesar del desconocimiento de muchos ancianos hacia las nuevas tecnologías, con la ayuda de supervisores expertos puede utilizarse estos dispositivos como recurso para la mejora de su condición física, salud mental y bienestar general ${ }^{10,11}$.

Por este motivo, resulta de gran interés plantear la utilización de videojuegos activos para el desarrollo de tal fin: mejorar la salud de nuestros mayores. En relación a este tipo de tecnología, trabajos como el de Alonso y Yuste $^{12}$, dictaminan que hasta hace poco esta tecnología se relacionaba con estereotipos asociados al sedentarismo y la inactividad social. Sin embargo, estudios posteriores han demostrado que si se respetan unos hábitos de juego, es decir, el tiempo dedicado, el entorno en el que se juega o el uso que se le da, puede resultar una actividad no sólo satisfactoria y segura, sino motivante ${ }^{6,13,14}$.

\section{LA TERCERA EDAD Y LA PRÁCTICA DE LA ACTIVIDAD FÍSICA}

Los hábitos sedentarios están cada vez más extendidos en los adultos mayores, los cuales, apenas realizan actividad física en comparación con otros sectores; pese a los beneficios que aporta a nivel físico, cognitivo y social ${ }^{15,16}$. En efecto, el hecho de que la tercera edad no realice actividad física puede deberse a falsos prejuicios sobre la concepción de la misma. Efectivamente, 
Pastor $^{17}$, destacó que para poder llevar a cabo la realización de ejercicio saludable en personas mayores, ha de plantearse la necesidad de convencer al anciano de sus aspectos positivos. Siendo necesario desmentir algunas de las creencias erróneas que poseen; siendo las principales que la vejez es sufrir enfermedades e incapacidades o que después de la jubilación lo bueno para la salud es descansar y no hacer nada ${ }^{5,9,15}$.

Estas premisas siguen la misma línea que el estudio realizado por Araújo y Manucussi $^{16}$, quienes afirman que el hecho de que nuestros mayores no realicen actividad física, puede deberse tanto a una discapacidad como a imposiciones culturales. Asimismo, determinan que las conductas más frecuentes en este sector son la pasividad y la inmovilidad, con un alto nivel de sedentarismo. No obstante, constataron que las mujeres mayores de 70 años constituían el perfil de ancianos más activo, predominando actitudes favorables hacia el ejercicio físico, ya sea por prescripción médica o iniciativa propia. Demostrándose por tanto, una disposición favorable de la tercera edad hacia un estilo de vida activo si se les promueve a ello ${ }^{15,18}$.

Por otro lado, resulta interesante citar los efectos positivos en los que se traduce la práctica de la actividad física. En este sentido, diversos estudios destacan a nivel fisiológico, la mejora del sistema cardiovascular, respiratorio, musculo-esquelético o del aparato locomotor ${ }^{5,16,19}$. Del mismo modo, a nivel psicológico, el ejercicio físico permite la mejora de la capacidad de atención, la memoria, el razonamiento, la estabilidad emocional o estados de ansiedad y depresión ${ }^{19,20}$. En esta línea, Correa et al. ${ }^{21}$ exponen que la participación en actividades físicas (aeróbicas y anaeróbicas), resulta positiva al reducir en un $25 \%$ todas las causas de mortalidad, además de mejorar la autoestima y generar un envejecimiento más saludable. Igualmente, estos autores atendieron al concepto de envejecimiento activo, entendiéndolo como estrategia para optimizar la calidad de vida, las oportunidades de salud y la participación comunitaria de la población adulta mayor ${ }^{2,5}$. Por otro lado, el estudio realizado por Méndez y Fernández ${ }^{22}$ concluye que el ejercicio físico regular puede producir beneficios significativos para la salud, 
independientemente de la edad y capacidades de los participantes; aumentando la esperanza de vida y reduciendo las posibles deficiencias funcionales vinculadas al paso del tiempo. Por ello, la actividad física permite a las personas mayores prevenir enfermedades crónicas, mantener un estado de vida independiente y mejorar la calidad de la vejez ${ }^{2,15,23}$.

La importancia de seguir un estilo de vida activo cae por el propio peso de los beneficios que aporta. No obstante, siempre habrá prejuicios a la hora de realizar actividad física, como el tiempo atmosférico o la monotonía ${ }^{14}$. De hecho, Trujillo et al. ${ }^{14}$ manifiestan necesidad de innovar las prácticas tradicionales que se adhieren a actividades mecánicas, aburridas y monótonas. Aquí es donde entran en juago las nuevas tecnologías, y es que remitiéndonos a la afirmación de Bosley ${ }^{9}$, los videojuegos representan un gran recurso a explotar en la mejora de la calidad de vida de nuestros mayores.

\section{LA TERCERA EDAD Y LOS EXERGAMES}

El concepto "Exergames" proviene de la unión de dos palabras: EXERCISE (Ejercicio) y GAMES o GAMING (Juegos). De este modo, este término hace referencia a aquellos videojuegos que pueden ser jugados con el movimiento corporal, sin necesidad de usar mandos con botones ${ }^{24}$. De hecho, autores como Sinclair y Hingston ${ }^{25}$, acuñan esta definición, afirmando que el empleo de videojuegos activos puede hacer más divertida y motivante la práctica de actividad física en multitud de contextos.

Por tanto, podemos decir que los "Exergames" surgen como una nueva forma de realizar ejercicio, en contraposición de las prácticas monótonas y aburridas más tradicionales ${ }^{14}$. De hecho, varias investigaciones definen los videojuegos activos como aquellos que permiten la realización de actividad física a través de la simulación de diferentes entornos y realidades ${ }^{24,25}$. De la misma manera, Trujillo et al. ${ }^{14}$ concretaron varios estudios sobre el empleo de "Exergames" en personas de edad avanzada, emulando diferentes modalidades deportivas 
(golf, tenis, fútbol, etc.), a través de los cuales se hallaron resultados positivos, fruto de la combinación entre el ejercicio físico y el entretenimiento.

En torno a las posibilidades de uso de este tipo de videojuegos, existen múltiples estudios que emplean los "Exergames" con diferentes finalidades en muestras de diversa edad y contexto, concretándolos como instrumentos no sólo para la práctica de actividad física desde la comodidad del hogar, sino como respuesta terapéutica. Claros ejemplos son los trabajos realizados por Gatica et al. ${ }^{26}$, Brown ${ }^{27}$ o Brumels et al. ${ }^{28}$, quienes utilizaron estos dispositivos, y más concretamente la plataforma Nintendo Wii, para el desarrollo del equilibrio en adultos mayores y la mejora de la ergonomía postural. En esta línea, Mombarg et al. ${ }^{29}$ ampliaron su estudio al entrenamiento del equilibrio en niños con problemas de índole motor, alcanzando resultados positivos mediante el empleo de videojuegos activos.

Otras investigaciones de gran interés han sido las realizadas en la parcela del sistema nervioso central, abarcando daños cerebrales y en la médula espinal. Por ejemplo, Deutsch et al. ${ }^{30}$ utilizaron la plataforma Wii con el objetivo de mejorar el control motor de un adolescente con parálisis cerebral, obteniendo una mejoría en el control postural y la movilidad funcional. Igualmente, Klompstra et al. ${ }^{31}$ estudiaron los beneficios del entrenamiento con este tipo de videojuegos en ancianos con posibilidad de sufrir cardiopatías, obteniendo resultados positivos para su prevención.

Mencionados algunos de los efectos positivos a nivel físico que supone el uso de este tipo de dispositivos, caben destacar aquellos que operan a nivel cognitivo, emocional y social. Dichos beneficios cobran forma en números estudios como el de Rosenberg et al. $^{32}$, quienes dictaminaron que los "Exergames", tienen también una repercusión positiva en la intervención contra la depresión. En esta línea, Sinclair y Hingston ${ }^{25}$ destacaron la importancia de la adecuada concreción de los efectos visuales, la interfaz de actuación o la tarea a realizar, como elemento sustancial para evitar el aburrimiento en adultos mayores. Asimismo, en el ámbito motivacional, Brox et al. ${ }^{6}$ defendieron 
la hipótesis de que los "Exergames" pueden ser utilizados para motivar y persuadir a nuestros mayores a hacer más ejercicio, simplemente porque es divertido ${ }^{33}$.

Poniendo en relieve los múltiples beneficios que implica la utilización de videojuegos activos en la tercera edad, parece sensato pensar que su empleo pueda mejorar la salud, la sensación de bienestar, y por tanto, calidad de vida. Con esta tecnología podrían mejorar su condición física (incluyendo el desarrollo de sistemas orgánicos y la prevención de enfermedades, como se ha destacado anteriormente) y el estado anímico (como la concreción de estados mentales equilibrados generados a través de la práctica de actividad física).

\section{CONCLUSIONES}

Se ha definido el contexto de la sociedad actual, la cual, da pasos de gigante tecnológicamente hablando. Una sociedad que se define claramente por la interconectividad y por el afán de acumular información. También, vemos que otra de las características más importantes que la configuran, al margen de la tecnología, es el creciente número de personas mayores; consecuencia directa del aumento de la esperanza de vida y la disminución de la natalidad.

Sin embargo, que haya más personas mayores y que estemos en una sociedad tecnológicamente avanzada, no significa que este sector goce de una buena calidad de vida hablando en términos de salud. Ya que como se ha mencionado antes, muchos de nuestros mayores no practican ningún tipo de actividad física que permita la mejora de su salud y la prevención de enfermedades.

De esta premisa es de donde surge el planteamiento de usar la tecnología que nos rodea, concretada en los videojuegos activos o "Exergames", proponiendo su implementación en programas de intervención que ayuden a nuestros 
ancianos a mejorar su condición físico-mental, y con ello, lograr estados de bienestar en edades avanzadas.

\section{REFERENCIAS BIBLIOGRÁFICAS}

1. Eguía JL, Contreras RS, Solano L. Videojuegos: conceptos, historia y su potencial como herramientas para la educación. 3C TIC. 2012; 1(2): 114.

2. Brigeiro M. "Envejecimiento exitoso" y "tercera edad": problemas y retos para la promoción de la salud. Investigación y Educación en Enfermería. 2005; 23(1): 102-109.

3. Forrellat-Barrios M, Hernández-Hernández A, Guerrero-Molina $Y$, Domínguez-López Y, Naranjo-Cobas Al, Hernández-Ramírez $\mathrm{P}$. Importancia de los ejercicios físicos para lograr una ancianidad saludable. Rev Cubana Hematol, Inmunol Hemoterap. 2012; 28(1): 3440.

4. Carone G, Costello D. ¿Llega Europa a la tercera edad?: la UE debe tomar en serio las proyecciones recientes que indican que el envejecimiento de la población tendrá un gran impacto económico y presupuestario. Finan \& Des. 2006; 43(3): 28-31.

5. Geis PP. TERCERA EDAD. Actividad física y salud. Barcelona: Editorial Paidotribo; 2001.

6. Brox E, Luque LF, Evertsen GJ, Hernández JE. Exergames for elderly: Social exergames to persuade seniors to increase physical activity. 5th International Conference on Pervasive Computing Technologies for Healthcare and Workshops. 2011; May 23 - May 26. Dublín. IEEE; 2011. 546-549.

7. Dussel I, Quevedo LA. Educación y nuevas tecnologías: Los desafíos pedagógicos ante el mundo digital. Buenos Aires: Fundación Santillana; 2010.

8. Sevilla M, Salgado MC, Osuna, NC. Repercusión en el desempeño escolar de los adolescentes con el uso de las Tecnologías de Información y Comunicación. Rev lb Produc Academ Gest Educ. 2015; 2(1): 1-19.

9. Bosley S. It Can Change Your Life. Adult Learn. 2011; 22(8): 28-30.

10. Muñoz JE, Henao OA, López JF. Sistema de Rehabilitación basado en el Uso de Análisis Biomecánico y Videojuegos mediante el Sensor Kinect. Rev Tecnologic. 2013; 43-54.

11. Thin AG, Poole N. Dance-based exergaming: User experience design implications for maximizing health benefits based on exercise intensity and perceived enjoyment. Transact Edutainm. 2010; 4: 189-199.

12. Alonso DL, Yuste TR. Teorías de la educación de adultos que subyacen en el uso de videojuegos. EKS. 2015; 15(4): 160-183.

13. Morillas A, Martínez G. La influencia de las nuevas tecnologías: videojuegos, redes sociales e internet, en los consumidores seniors en España. I Congreso Internacional de Comunicación y Sociedad Digital. 2013; May 5. La Rioja, España. UNIR; 2013. 1-28. 
14. Trujillo J, Muñoz J, Villada J. Exergames: una herramienta tecnológica para la actividad física. Rev Med Risarald. 2013; 19(2): 126-130.

15. Jiménez $Y$, Núñez $M$, Coto $E$. La actividad física para el adulto mayor en el medio natural. InterSedes. 2013; 14(27): 168-181.

16. Araújo CL, Manucussi e Faro AC. La práctica de actividad física en personas mayores del Valle del Paraíba, São Paulo, Brasil. Enferm Glob. 2012; 11(28): 204-212.

17. Pastor JL. Tercera edad, actividad física y estado de bienestar. REEFD. 2010; (3): 1-10.

18. Fontana RJ, Grasso L, Pinto MF. Beneficios percibidos por adultos mayores que asisten a un programa de actividad física. VI Congreso Internacional de Investigación y Práctica Profesional en Psicología XXI. 2014. Buenos Aires, Argentina. UBA; 2014. 93-96.

19. López GF, López L, Díaz A. Efectos de un programa de actividad física en la condición física de escolares con TDAH. Rev lb CC Act Fís Dep. 2014; 3(3): 24-37.

20. Barriopedro M, Mallol L, Eraña de Castro I. Relación de la actividad física con la depresión y satisfacción con la vida en la tercera edad. Rev Psicol Dep. 2001; 10, 239-246.

21. Correa JE, Gámez ER, Ibánez MI, Rodríguez KD. Aptitud física en mujeres adultas mayores vinculadas a un programa de envejecimiento activo. Rev Salud Uis. 2011; 43(3): 263-270.

22. Méndez A, Fernández J. Prescripción de la actividad física en personas mayores: recomendaciones actuales. REEFD. 2010; 3: 20-28.

23. Petrovic M, Ruiz-Montero PJ. Transformación antropológica en mayores con diabetes tipo II a través del ejercicio: Pilates y ejercicio aeróbico. Rev Ib CC Act Fís Dep. 2015; 4(1): 1-5.

24. Beltrán VJ, Valencia A, Molina JP. Los videojuegos activos y la salud de los jóvenes: revisión de la investigación. Rev Int Med Cienc Act Físic Dep. 2011; 10(41): 203-219.

25. Sinclair J, Hingston $P$, Masek $M$. Considerations for the design of exergames. Proceedings of the 5th international conference on Computer graphics and interactive techniques in Australia and Southeast Asia. 2007; Dec 1 - Dec 4. Perth, Western Australia. AMC; 2007. 289296.

26. Gatica V, Elgueta E, Vidal C, Cantin M, Fuentealba J. Impacto del entrenamiento del balance a través de realidad virtual en una población de adultos mayores. Int J Morphol. 2010; 28(1): 303-308,

27. Brown R, Sugarman H, Burstin A. Use of the Nintendo Wii Fit for the Treatment of Balance Problems in an Elderly Patient with Stroke: A Case Report. Int J Rehabil Res. 2009; 32: 109-110.

28. Brumels K, Blasius T, Cortright T, Oumedian D, Solberg B. Comparison of efficacy between traditional and video game based balance programs. J Americ Kinesiother Associat. 2010; 62(4): 26-31.

29. Mombarg R, Jelsma D, Hartman E. Effect of Wii-intervention on balance of children with poor motor performance. Research Develop Disab. 2013; 34(9): 2996-3003. 
30. Deutsch JE, Borbely M, Filler J, Huhn K, Guarrera Bowlby P. Use of a Low-Cost, Commercially Available Gaming Console (Wii) for Rehabilitation of an Adolescent With Cerebral Palsy. Phys Ther. 2008; 88(10): 1196-1207.

31. Klompstra LV, Jaarsma T, Strömberg A. Exergaming in older adults: A scoping review and implementation potential for patients with heart failure. European J Cardiov Nurs. 2013; 13(5): 388-398.

32. Rosenberg D, Depp C, Vahia IV, Reichstadt J, Palmer BW, Kerr J, Norman G, Jeste DV. Exergames for subsyndromal depression in older adults: a pilot study of a novel interaction. Americ J of Geriatric Psychiat. 2010; 18(3): 221-226.

33. Reigal RE, Videra A. Frecuencia de práctica física y autoeficacia en la tercera edad. Revista Internacional de Medicina y Ciencias de la Actividad Física y del Deporte. 2013; 13(49): 107-120.

Referencias totales citadas: 33

Referencias citadas correspondientes a la Rev Ib CC Act Fis Dep: 2 